\title{
Performance Analysis of Model-Based Localization of High-Frequency Acoustic Sources in 3D
}

\author{
Ehsan Zamanizadeh, João Gomes \\ Institute for Systems and Robotics \\ Instituto Superior Técnico (IST) \\ Technical University of Lisbon (UTL) \\ 1049-001 Lisbon, Portugal \\ Email: \{ezamanizadeh, jpg $\} @$ isr.ist.utl.pt
}

\author{
José M. Bioucas-Dias \\ Instituto de Telecomunicações \\ Instituto Superior Técnico (IST) \\ Technical University of Lisbon (UTL) \\ 1049-001 Lisbon, Portugal \\ Email: jose.bioucas@1x.it.pt
}

\author{
Ilkka Karasalo \\ Aeronautical and Vehicle Engineering \\ Marcus Wallenberg Laboratory \\ KTH Royal Institute of Technology \\ SE-100 44 Stockholm, Sweden \\ Email: ilkkak@kth.se
}

\begin{abstract}
A robust model-based source localization (MBSL) scheme for 3D positioning of high-frequency underwater acoustic (UWA) transmitters, as well as performance assessment of 3D speed vector estimation of the acoustic source, deploying two linear receiver arrays, is developed and evaluated. In addition, this paper presents the sensitivity evaluation of MBSL to uncertainties involved in environmental parameters, including the effects of sound speed profile and bathymetry mismatches. The results presented here pertain to the general framework of inferring side information concerning an UWA link from the environmental signature imprinted on waveforms by the propagation medium. The presented algorithms directly operate on estimated linear time-varying UWA channel responses, represented as 3-dimensional delay-Dopplerdepth functions (DDDF). Estimation of DDDF coefficients is achieved jointly for all receivers deployed at different depths using Basis-Pursuit (BP) tools, which can efficiently handle sparse unconstrained $\ell_{2}-\ell_{1}$ minimization, and directly operate on the complex signals of baseband models. To extract delay/Doppler information related to each path from the DDDF representation, an efficient energy-based scheme is developed to detect and label wavefronts using clustering techniques inspired by image processing.
\end{abstract}

\section{INTRODUCTION}

Ocean acoustic tomography (OAT) is developed based on the fact that environmental variables like sound speed profile, source/receiver configuration, water depth, and bottom structure imprint a particular signature on acoustic signals, in a way that some of these characteristics can be recovered by applying inverse problem techniques. The underwater medium poses significant challenges to OAT due to the complexity of sound propagation resulting from the nonhomogeneous sound speed, multiple interactions with the sea surface and bottom, and Doppler-induced compression or expansion of signals [1].

Much of the published work on underwater source localization is concerned with low-frequency sources, where interactions with poorly-characterized bottom sediment layers make for a difficult estimation problem. Matched-field processing (MFP) has been quite successful at accurately localizing sources at frequencies up to several hundreds of $\mathrm{Hz}$ even in environments with strong refraction, strong bottom interactions, or markedly non-flat bathymetry [2]. Unlike traditional ocean acoustic tomography (OAT) approaches, whose frequencies are much smaller than those found in underwater communication systems, our designed scheme acquires position and velocity information from communication waveforms, where full-field inversion methods have limited use both due to large modeling uncertainties and high required computational loads.

We present a robust technique to efficiently localize acoustic sources and estimate their relative speeds in 3D space based on matching predicted and observed arrivals in channel responses obtained at 2 arrays of receivers, as a by-product of highfrequency UWA communications.

The proposed 3D localization and speed estimation scheme could be divided into four main stages. The first stage, sparse channel estimation and clustering, aims to provide accurate estimates of channel responses using Basis Pursuit (BP) methods and characterize the main propagation paths in the channel. The second stage, wavefront labeling, cooperates with the third stage, UWA source localization, to assign an appropriate number of bounces (i.e., a label) to each detected wavefront. Using the extracted delay information from labeled wavefronts, estimation algorithms in the third stage provide accurate values for the source position. Finally, using the extracted Doppler shifts from the labeling stage and the estimated source position provided by MBSL, the source/receiver relative velocity vector is estimated.

Considering the uncertainties involved in the environmental parameters, this work also aims to analyze the robustness and sensitivity of our MBSL approach, namely, its sensitivity to model mismatches in sound speed profile, bathymetry and vertical linear array (VLA) position.

The paper comprises the following sections. Section II briefly presents the $\ell_{2}-\ell_{1}$ BP techniques used for sparse channel estimation. TDOA-based 3D source localization is outlined in Section III. Section IV concisely explains the procedure of wavefront segmenting and labeling for detecting and clustering wavefronts from channel estimates in 3D images. In section $\mathrm{V}$ we derive the 3D velocity vector of a UWA source using the Doppler information extracted in Section IV and 


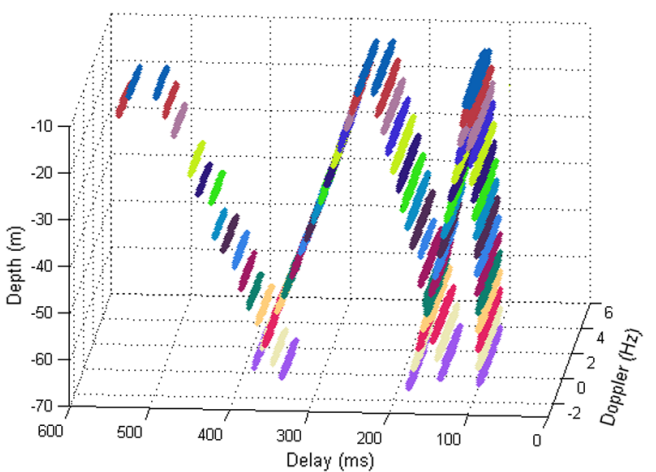

(a)

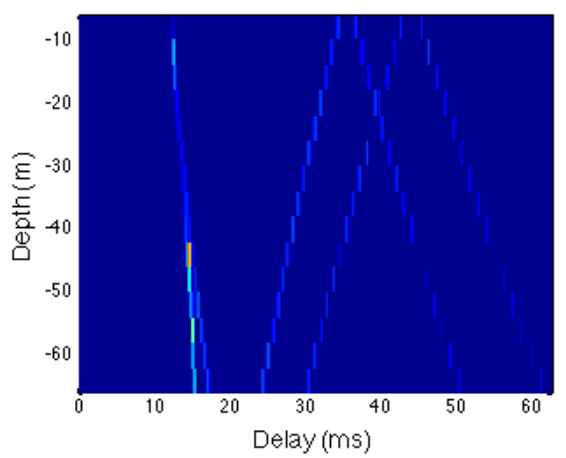

(b)

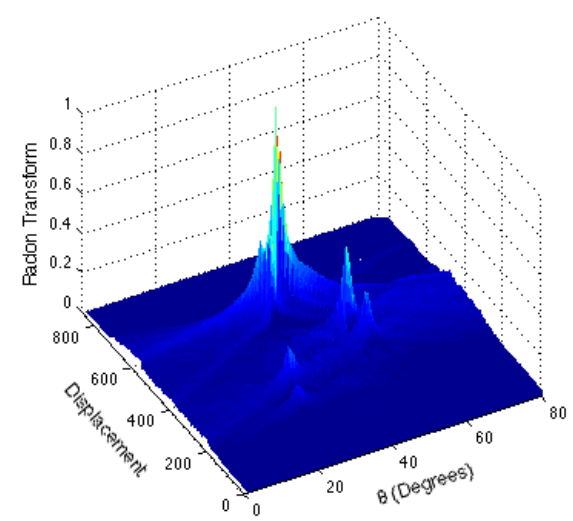

(c)

Fig. 1: (a) A sample DDDF for an array of 16 hydrophones with equal spacing. (b) Arrival time delay vs. receiver depth. (c) Radon transform of image $1 \mathrm{~b}$.

the final source position from Section III. The evaluation of MBSL sensitivity considering various sources of uncertainty is presented in section VI. Section VII provides numerical results on the performance of 3D source localization and speed estimation, using simulated data. Finally, Section VIII provides conclusions and outlines future perspectives.

Notation: Superscripts $(\cdot)^{T},(\cdot)^{H}$ stand for transpose and conjugate transpose (hermitian), respectively. $\ell_{p}$ norms are denoted by $\|\cdot\|_{p}$, and $\ell_{2}$ is assumed when the argument $p$ is omitted.

\section{Sparse UWA Channel Estimation}

Estimation of sparse time-varying channels through basis pursuit (BP) techniques is briefly reviewed here [3]. In this paper, the channel output is viewed as a sum of replicas of the input signal, each associated with a given delay, $\tau$, and Doppler shift, $\nu$, that are assumed constant over an averaging interval [4]. We adopt a linear time-varying model for UWA channels, representing them by their DDSFs [5]. We consider the following discrete-time model with input $x(n)$ and output $y_{m}(n)$ observed at the $m$-th array hydrophone

$$
y_{m}(n)=\sum_{k, l} u_{m, k, l} x_{l}(n-k), \quad x_{l}(n)=x(n) e^{j 2 \pi \nu_{l} n},
$$

where $\nu_{l}=\frac{l}{T f_{s}}$ is the $l$-th Doppler shift under sampling frequency $f_{s}$ and input block duration $T$.

Taking advantage of the linearity in DDSF coefficients, the channel model can be represented for all receivers in matrix form as

$$
\mathbf{Y}=\mathbf{X U}
$$

where $\mathbf{Y}$ denotes a matrix of observed samples for all receivers (one column per hydrophone), $\mathbf{U}$ defines the matrix of unknown DDDF coefficients, and $\mathbf{X}$ is the dictionary matrix built from a block of known transmitted samples. Sparse estimation of DDDF coefficients is derived by solving

$$
\min _{\mathbf{U}} \frac{1}{2}\|\mathbf{Y}-\mathbf{X U}\|_{2}^{2}+\tau\|\mathbf{U}\|_{1}
$$

using BP tools. While the first term $\left(\ell_{2}\right)$ quantifies the leastsquares fit between observed and predicted channel responses, the second one $\left(\ell_{1}\right)$ is a sparsity promoting regularizer that drives to zero insignificant channel coefficients [3].

\section{UWA 3D Source Positioning}

A two-step approach, exploiting the temporal and spatial structure of multipath observed at an array of sensors, was developed to determine the source position [6]. The first step, "Coarse Source Localization (CSL)", is designed based on free-space localization from time differences of arrival, in addition to configuration parameters for the acoustic link such as hydrophone positions, sound speed profile, and bathymetry, [7], [8] to provide a good initial estimate of the UWA transmitter with no a priori knowledge of its location and only crude environmental information. The second stage, "ModelBased Source Localization (MBSL)", improves the estimated source position by matching the predicted vs. observed pattern of wavefronts impinging upon the array.

For a single linear array (Figure 2), the direction of received signal and velocity vector component orthogonal to the vertical plane containing the source and receivers does not impact the DDDF. Therefore the proposed localization and speed estimation approaches are just able to estimate the communication range, source depth and the projection of velocity vector on source-receivers plane. To fully estimate the $3 \mathrm{D}$ position and velocity vector, at least two arrays of receivers would be needed to create a nonplanar configuration (Figure 3a).

\section{A. Coarse Source Localization (CSL)}

The method presented in this section estimates the source position, which is needed for model-based localization when multiple maxima/minima exist in the associated cost function that matches observed vs. predicted TDOA values. The method builds upon a localization algorithm for free-space propagation based on distance differences of arrival, which we modify to include some of the effects present in ocean waveguides. 


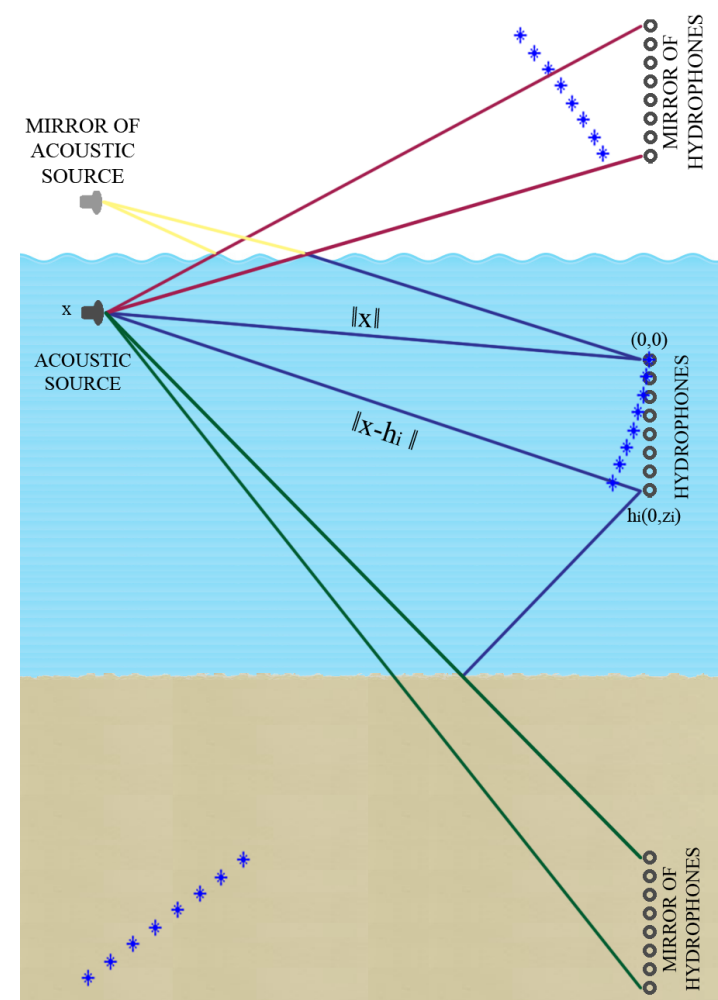

Fig. 2: Decomposition of the physical array into virtual surface and bottom-reflected images. Each arrival is matched to the propagation delay between the source and the associated image hydrophone.

The method we use is presented in [7], and matches squared range differences according to the cost function

$$
\min _{\mathbf{x}_{s}} \sum_{m=1}^{M}\left(\left\|\mathbf{x}_{s}-\mathbf{s}_{m}\right\|^{2}-\left(d_{m}+\left\|\mathbf{x}_{s}\right\|\right)^{2}\right)^{2} .
$$

where $\mathbf{x}_{s}$ and $\mathbf{s}_{m}$ denote the positions of the unknown source and the $m$-th sensor, and $d_{m}$ is the measured difference between the range from the source to sensor $\mathbf{s}_{m}$, and the range from the source to the reference sensor, located at the origin of the coordinate system. A fast iterative algorithm is proposed in [7] to efficiently find the global minimum of (4), even though, in general, this problem is not convex.

Since the localization scheme in [7] assumes free-space propagation, some adaptations are considered to account for the presence of multiple wavefronts in an ocean waveguide. We adopt the image method [1] to replace the physical array embedded in the waveguide with multiple virtual images in free space, associated with surface and bottom reflections as depicted in Figure 2 for a single array. To derive a reliable source position in $3 \mathrm{D}$, two linear arrays are deployed as it is illustrated in Figure 3a. To derive range differences from the source to the array hydrophones, the wavefront segmentation methods described in Section IV are first used to estimate delay differences. Multiplying theses time delays, $\tau_{m}$, by an average sound speed, $c_{m}$, provides distance differences to plug

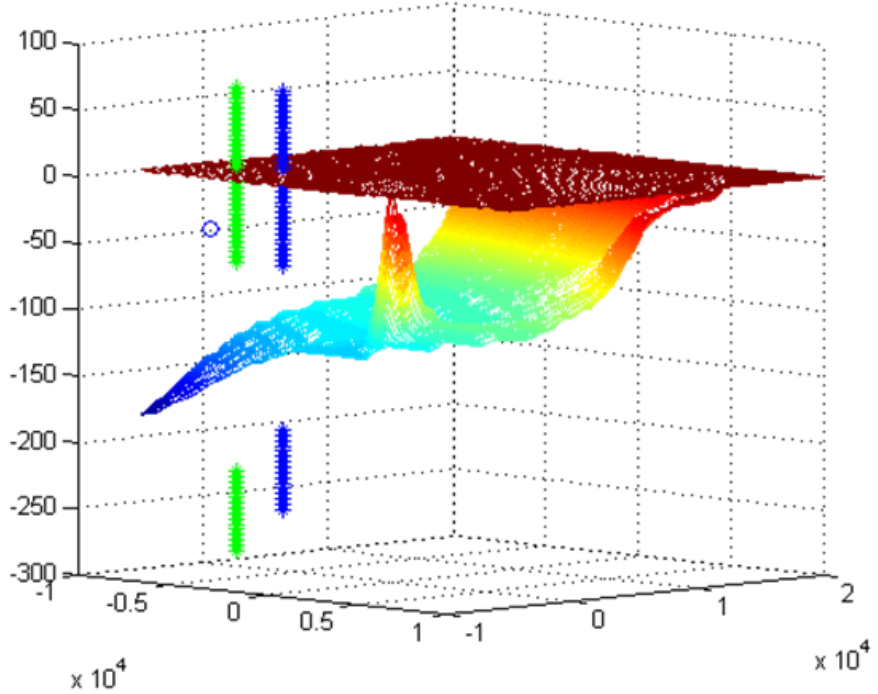

(a)

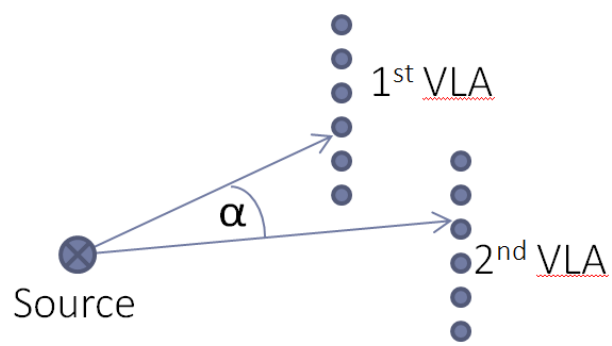

(b)

Fig. 3: (a) Decomposition of the 2 physical arrays into virtual surface and bottom-reflected images in 3D. (b) Source-arrays configuration with direction difference angle $\alpha$.

into (4), $d_{m}=c_{m} \tau_{m}$. We measure all delays relative to the earliest arrival (direct path) in the topmost hydrophone of one of the linear arrays.

To improve the accuracy of the proposed CSL approach several modifications are developed to adapt it to nonhomogeneous underwater waveguides [8]: (i) Considering nonlinearity in the sound speed profile, (ii) compensating for sloped bathymetry, (iii) compensating for acoustic ray bending.

1) Non-linearity in the Sound Speed Profile: Since the source position is unknown, the CSL technique can be used iteratively to gradually refine the mean velocities along each ray path. In the first iteration a single global average sound speed is used to compute an initial source position, and in subsequent iterations the most recent estimate $z_{s}$ is used. Similarly to the receiver array, the sound speed profile (SSP) is expanded into surface and bottom-reflected images for computing average sound speeds. This is shown in Figure 4c.

2) Compensating for Sloped Bathymetry in CSL: Unlike the basic CSL method depicted in Figure 2, which assumes flat (horizontal) bathymetry, in real scenarios the bottom could have any shape. For 2D positioning, the bathymetry could be 

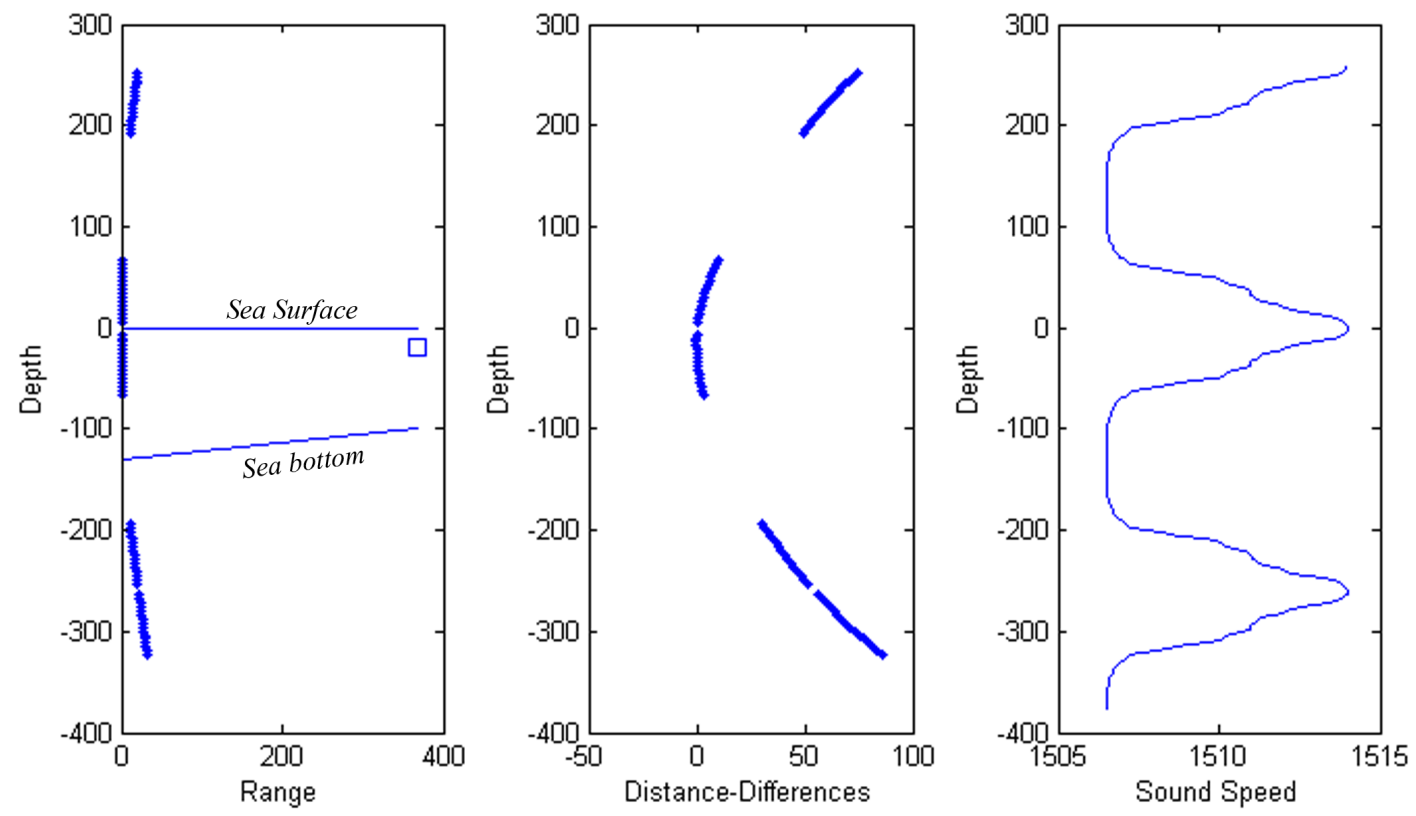

Fig. 4: (a) Physical array and its surface, bottom, surface-bottom and bottom-surface reflections with sloped bathymetry. The source is represented by a square on the right side. (b) Range differences to each hydrophone, relative to the source-to-reference range (topmost physical hydrophone). (c) Expanded sound speed profile for surface, bottom, surface-bottom and bottom-surface reflections.

approximated by multiple linear segments. With a piecewiselinear bottom, an iterative scheme is needed to determine the appropriate normal for each bottom reflection based on the current source position estimate. For a single segment (constant slope) the required modification for 2D positioning is depicted in Figure 4.

In 3D localization the line with constant slope is replaced by a plane defined by 3 points on the sea bottom. The best choices for these 3 points are the water depths at the locations of the linear arrays and the source. As the latter is unknown, an iterative scheme is applied to account for the water depth at the source position. Taking the average of the water depths of both linear arrays could provide a good initial guess for the water depth at the source position.

Our results suggest that only two CSL iterations are enough to reach a solution with sufficiently good quality to initialize the model-based localization scheme described in Section III-B.

3) Compensating for Acoustic Ray Bending: Due to variations in sound speed, acoustic rays do not travel along straight paths, whereas the basic CSL criterion (4) is built under the assumption that travel times are proportional to Euclidean distance (i.e., straight paths, constant speed). The CSL method should account for changes in mean sound speed when translating TDOAs into range differences, but also for the departure of ray trajectories from the straight line assumption. For compensating either of these distortions we resort to iterative processing, using the previous source position estimate to determine the required corrections, and then recomputing those coordinates [6]. This compensation procedure is more effective at ranges on the order of $1 \mathrm{~km}$ and higher, where ray bending becomes significant.

\section{B. Model-based Source Localization}

This section outlines the previously proposed MBSL scheme with some modifications to account for 3D positioning of the acoustic transmitter and 2 linear receiver arrays. The MBSL step refines the source position, previously estimated by CSL, matching the predicted vs. observed pattern of wavefronts impinging upon the array.

The proposed cost function penalizes the Euclidean norm of predicted vs. observed TDOA differences for all hydrophones and wavefronts for both linear arrays [6], [9]. We iteratively solve

$$
\min _{\mathbf{x}_{s}} f\left(\mathbf{x}_{s}\right)=\left\|\boldsymbol{\tau}_{1}-\hat{\boldsymbol{\tau}}_{1}\left(\mathbf{x}_{s}\right)\right\|^{2}+\left\|\boldsymbol{\tau}_{2}-\hat{\boldsymbol{\tau}}_{2}\left(\mathbf{x}_{s}\right)\right\|^{2},
$$

where, for $i \in\{1,2\}, \boldsymbol{\tau}_{i}$ denotes the vector of actual TDOAs, obtained by segmenting and classifying the observed wavefronts for linear array $i$, as described in Section IV, and $\hat{\boldsymbol{\tau}}_{i}\left(\mathbf{x}_{s}\right)$ is the corresponding vector of TDOAs obtained from the acoustic ray tracer for a source located at $\mathrm{x}_{s}$.

Specifically, in each iteration of the minimization algorithm we update the current source position estimate by solving the linear LS problem

$$
\mathbf{x}_{s, k+1}=\arg \min _{\mathbf{x}_{s}}\left(\left\|\boldsymbol{\tau}_{1}-\hat{\boldsymbol{\tau}}_{1, k}\left(\mathbf{x}_{s}\right)\right\|^{2}+\left\|\boldsymbol{\tau}_{2}-\hat{\boldsymbol{\tau}}_{2, k}\left(\mathbf{x}_{s}\right)\right\|^{2}\right),
$$




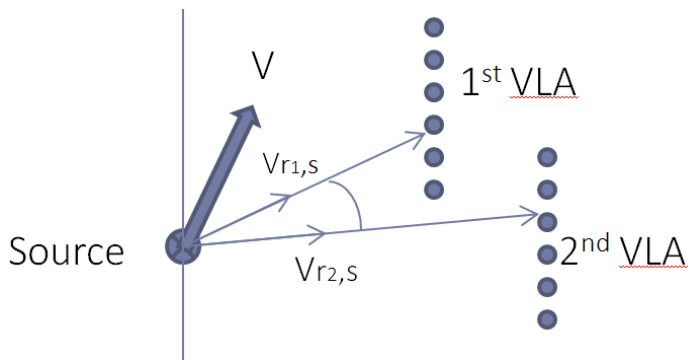

(a)

Fig. 5: (a) Projection of speed vector along the transmitter/receiver path.

where the nonlinear mapping from source position to delay differences is replaced by a first-order approximation $\hat{\tau}_{i}\left(\mathbf{x}_{s}\right)$ for $i \in\{1,2\}$ around the current source position estimate $\mathbf{x}_{s, k}$ as

$$
\hat{\boldsymbol{\tau}}_{i, k}\left(\mathbf{x}_{s}\right) \triangleq \hat{\boldsymbol{\tau}}_{i}\left(\mathbf{x}_{s, k}\right)+D \hat{\boldsymbol{\tau}}_{i}\left(\mathbf{x}_{s, k}\right)\left(\mathbf{x}_{s}-\mathbf{x}_{s, k}\right),
$$

and $D \hat{\boldsymbol{\tau}}_{i}\left(\mathbf{x}_{s, k}\right)$ denotes the Jacobian matrix of the delay vector for array $i$ (see [6] for details). For $M$ sensors and $N$ wavefronts $\hat{\boldsymbol{\tau}}_{i} \in \mathbb{R}^{M N}$ and $D \hat{\boldsymbol{\tau}}_{i} \in \mathbb{R}^{M N \times 2}$. The final source position is obtained when the solution satisfies a stopping criterion.

\section{WAVEFRONT Detection AND LABELING}

Detecting wavefronts as a whole across the array provides much more robust detection and classification performance than would be possible by independently processing individual hydrophone signals. To detect and segment the wavefronts from a DDDF (figure 8) and extract delay/Doppler information related to each propagation path from the DDDF representation, a robust method is developed using voting-based image processing tools for line detection [8].

To reduce the computational complexity, we search for wavefronts in projections of the DDDF onto the delay-depth and Doppler-depth planes. A simple parametrization for a delay-depth planar wavefront contains the angle of arrival relative to the array axis, $\phi$. For a uniform linear array the candidate delay at the $m$-th sensor for this hypothetical wavefront would be

$$
\tau_{m}(\tau, \phi)=\tau+(m-1) \frac{d}{c} \sin \phi,
$$

where $d$ is the intersensor separation and $c$ is the mean sound speed. We evaluate the wavefront energy metric for these parameters, $J(\tau, \phi)$, as

$$
J(\tau, \phi)=\sum_{m=1}^{M}\left|U_{m}\left(\tau_{m}, \cdot\right)\right|,
$$

where $\left|U_{m}(\tau, \cdot)\right|$ denotes the magnitude of the $m$-th DDSF accumulated over all Doppler frequencies for any particular $\tau$. In practice we consider approaches based on the Radon and Fourier transforms to efficiently evaluate this cost (see Figures $1 \mathrm{~b}-1 \mathrm{c})$. Given the wide disparity of wavefront amplitudes we use the following successive cancellation approach to sequentially detect them: (i) Find the maximum of (9) and store the corresponding wavefront parameters. (ii) Remove the effect of the detected wavefront by applying a zero mask to $\left|U_{m}(\tau, \cdot)\right|$ in a narrow strip along the wavefront direction/delay. (iii) Recompute the energy metric for the masked DDSF and return to the first step until the residual energy is sufficiently low. Projected (2D) wavefront detection, yields a set of slices in delay-Doppler-depth space, orthogonal to the Doppler axis. Then we project each slice onto the Doppler-depth plane, and find the remaining parameters to fully characterize the orientation of wavefronts depicted in Figure 8.

Each detected wavefront must be labeled according to the number of surface and bottom bounces, so that the corresponding delays can be assigned to the correct images of the array sensors for our localization criteria based on matching distances/delays. A practical labeling scheme that leverages the low computational cost of our CSL algorithm to examine multiple candidate hypotheses is developed in [10].

\section{Velocity Estimation of the Acoustic Source}

Velocity vector estimation of the acoustic source is achieved by matching the observed and predicted evolutions of Doppler shifts in 3D wavefronts. The proposed strategy requires a good estimate of source position to compute the departure angle of acoustic paths for each individual hydrophone in the receiver array [8].

The observed Doppler shift, $\Delta f$, is approximately given by

$$
\Delta f=-\frac{v_{s, r}}{c} f_{c},
$$

where $v_{s, r}$ is the projection of the relative velocity vector along the transmitter/receiver path of interest and $f_{c}$ is the carrier frequency. An iterative scheme described in [11] can be used to efficiently compute eigenrays. Alternatively, the same acoustic ray tracer used for MBSL directly yields the grazing angle associated with each source-receiver eigenray [8].

In UWA channels, we can take advantage of multipath to solve an overdetermined system of equations matching Doppler shifts for all hydrophones and paths (wavefronts). The velocity vector component orthogonal to the plane comprising the source and receivers does not impact the DDDF, and hence it is not detectable by a single linear array. To fully estimate the $3 \mathrm{D}$ velocity vector, at least two arrays of receivers would be needed to create a nonplanar configuration. Defining a matrix, $\mathrm{U}$ containing projection coefficients for all rays, we have:

$$
\mathbf{v}_{s, r}=\arg \min _{\mathbf{v}}\left\|\frac{f_{c}}{c_{z}} \mathbf{U} \mathbf{v}-\Delta \mathbf{f}\right\|
$$

where $\mathbf{v}_{s, r}$ is the velocity vector with $v_{x}, v_{y}$ and $v_{z}$ components, vector $\Delta \mathbf{f}$ contains the $\left(M_{1}+M_{2}\right) N$ Doppler shifts associated with $M_{i}$ sensors of array $i$ and $N$ wavefronts, and $c_{z}$ is the sound speed at the source depth.

The full iterative source localization and speed estimation procedure is as follows:

1) The UWA channel response is estimated using the basis pursuit method described in Section II. 


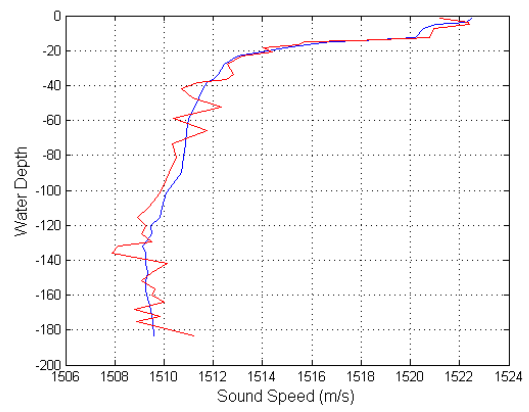

(a)

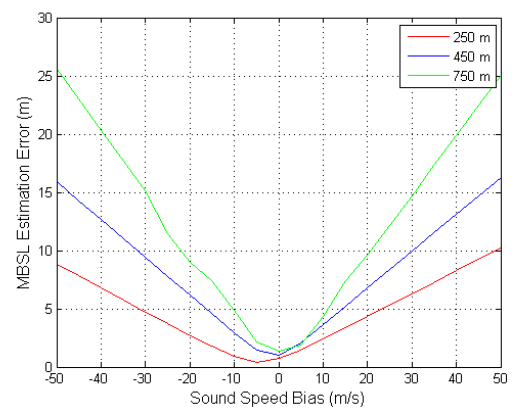

(b)

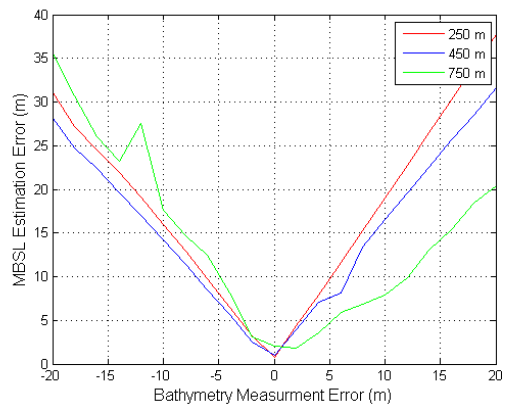

(c)

Fig. 6: Sensitivity Analysis of MBSL to Environmental Uncertainties. (a) Measured SSP and noisy SSP, respectively plotted in blue and red. (b) Sensitivity analysis of MBSL to SSP mismatch for various communication ranges. (c) Sensitivity analysis of MBSL to bias in batheymetry profile for various communication ranges.

2) Using segmentation methods from section IV, the TDOAs and Doppler shifts for all hydrophones and paths are computed.

3) The initial guess for source position to be used in MBSL is obtained by implementing the CSL scheme of Section III-A.

4) An acoustic channel model is used to predict the time delays for the current source location estimate. By computing the derivatives of time delay with respect to the acoustic source depth and communication range, we update source position by solving an overdetermined linear LS problem.

5) Using the Doppler shifts from section IV and estimated source position provided by MBSL in section III-B, the velocity vector of the source is estimated by solving (11).

\section{Sensitivity Analysis of MBSL to ENVIRONMENTAL UNCERTAINTIES}

This section investigates the dependency of uncertainty in the output of the acoustic localization model to different sources of uncertainty in the environmental variables (such as sound speed profile, bathymetry and array position).

The motivation for this study includes (i) evaluation of the robustness of the localization results in the presence of uncertainty in the inputs, (ii) better understanding of the interactions between environmental parameters and MBSL estimation results, (iii) improving the robustness by identifying variables that cause significant uncertainty in the localization output.

The speed of sound in water is a function of pressure, temperature and salinity. A sample measured sound speed profile (SSP) for CALCOM'10 sea trial is plotted in Figure 6a (in blue). To evaluate the sensitivity of the model-based localization technique to deviations in the SSP, measurement noise with variable power is added to each measured point (in red in Figure 6a). Applying Monte Carlo approach for 200 experiments for each case, negligible positioning error is observed at the MBSL output. This can be interpreted by the fact that the mean of SSP over depth remains constant for different noise powers, thus the sum of induced localization errors essentially cancels out.

The estimation error of MBSL output as a function of bias in SSP measurement for various communication ranges from 250 $\mathrm{m}$ to $750 \mathrm{~m}$ are presented in Figure 6b. For all cases, the source is deployed at a depth of $40 \mathrm{~m}$. The receiver array consist of 16 hydrophones with $4 \mathrm{~m}$ spacing. As the results illustrate, for all channel configurations, MBSL estimation output does not present high sensitivity to an acceptable measurement error in sound speed (less than $10 \mathrm{~m} / \mathrm{s}$ deviation). As expected, for higher uncertainty in SSP and higher communication ranges, we observe higher estimation error.

Figure $6 c$ provides the MBSL positioning error as a function of the bias in bathymetry profile for the same channel configurations as in Figure 6b. As results demonstrate, high sensitivity to discrepancy in water depth (bathymetry profile) is observed. When compared with the results for SSP, the trend as a function of mismatch is now less linear and less predictable. Since the MBSL is more sensitive to the bathymetry profile, high deviation in water depth measurements may cause the (iterative) algorithm to become trapped in undesirable local minima.

For predominantly flat bathymetry errors in VLA position will naturally affect the estimated source position by the same amount.

\section{Performance Assessment}

The performances of the proposed UWA source localization and velocity estimation approaches are assessed in 2D and 3D for single-carrier (QPSK) transmissions over simulated channels for various communication ranges and varying relative speed within the limit of 6 knots.

An UWA simulator, developed by the University of Algarve, is used to assess the proposed source localization and speed estimation methods in $2 \mathrm{D}$ and $3 \mathrm{D}$. In each simulation, a QPSK packet is transmitted at $2.4 \mathrm{kbaud}$, with $5.5 \mathrm{kHz}$ carrier 


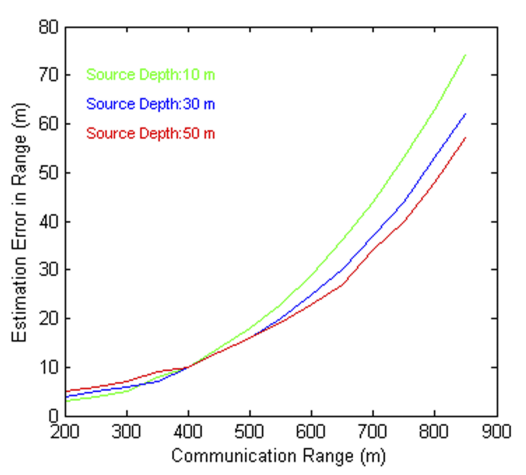

(a)

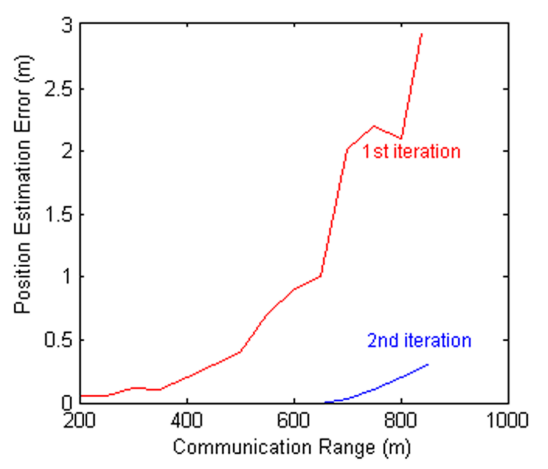

(b)

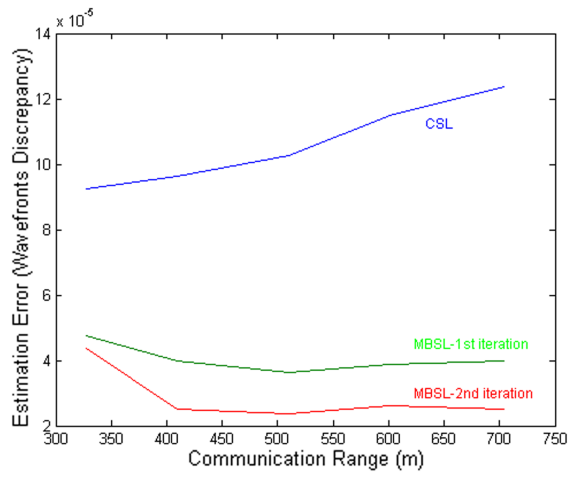

(c)

Fig. 7: Simulation and experimental results for source localization. (a) Simulation results for CSL. Range estimation error for different source depths. (b) Simulation results for MBSL with flat bathymetry. (c) Evaluation of CSL and MBSL methods with real CALCOM'10 data. The figure shows a global discrepancy metric of predicted vs. observed TDOAs.

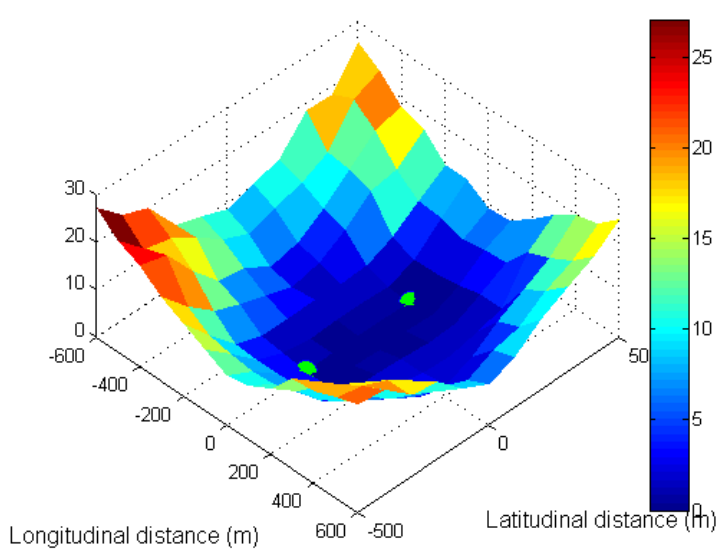

(a)

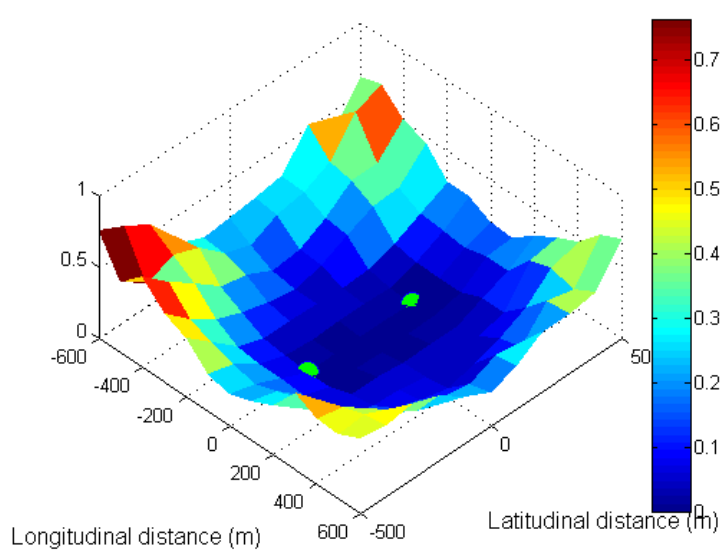

(b)

Fig. 8: Evaluation of source localization in 3D using simulation data. Two linear receiver arrays are located at $(0,200)$ and $(0,-200)$ which are shown by green circles. (a) CSL estimation error for various source positions (b) MBSL estimation error after two iterations for various source position.

frequency, $4.5 \mathrm{kHz}$ bandwidth, root-raised-cosine (RRC) pulse shape (88\% rolloff) for total duration of $3 \mathrm{~s}$ with a sandy bottom $\left(1600 \mathrm{~m} / \mathrm{s}, 2 \mathrm{~g} / \mathrm{cm}^{3}, 0.8 \mathrm{~dB} / \lambda\right)$. The baseband received signal is sampled at 4 times the symbol rate, $f_{s}=12 \mathrm{kHz}$. For 3D estimation, two linear arrays are considered with various source-array configurations. Each linear array consists of 16 hydrophones, placed at $4 \mathrm{~m}$ intervals between $6 \mathrm{~m}$ and $66 \mathrm{~m}$ depths.

Figure 7a shows results for range errors at three different source depths as a function of the actual source range. It is clear that errors increase with communication range. The norm of the source localization error obtained by MBSL, with CSL initialization, as a function of range (source depth is $30 \mathrm{~m}$ ) after the first and second iterations is presented in Figure $7 \mathrm{~b}$. As in CSL, errors grow larger as the communication range increases. However, given the good initial point, MBSL is able to drastically reduce the total source localization error within 2 iterations.

Figure 8a presents the CSL estimation error using two linear arrays in $3 \mathrm{D}$. The arrays are located at $(0,200)$ and $(0,-$ 200 ) and the localization error is obtained for various source positions with respect to the arrays. The acoustic source depth for all cases is constant and equal to $30 \mathrm{~m}$. Comparing the results with the 2D case in Figure 7a we can infer that we obtained more accurate results for the region between the two arrays. It is clear that, in general, larger estimation errors are obtained for longer communication ranges with respect to both arrays. This is to be expected, as the set of selected wavefronts carry less information about the source position as it moves further into the far-field of the receiver arrays.

Similar to Figure 8a, the results of MBSL for the same configurations are shown in Figure 8b. Note that there is 


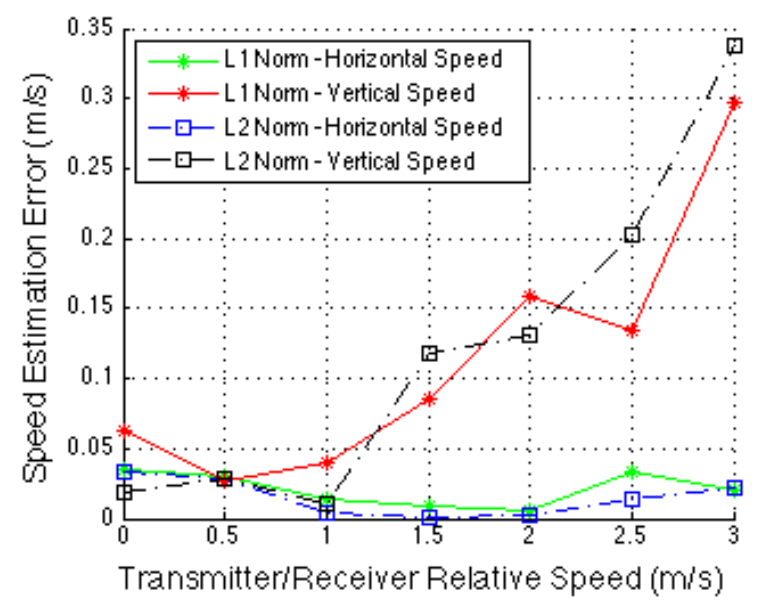

(a)

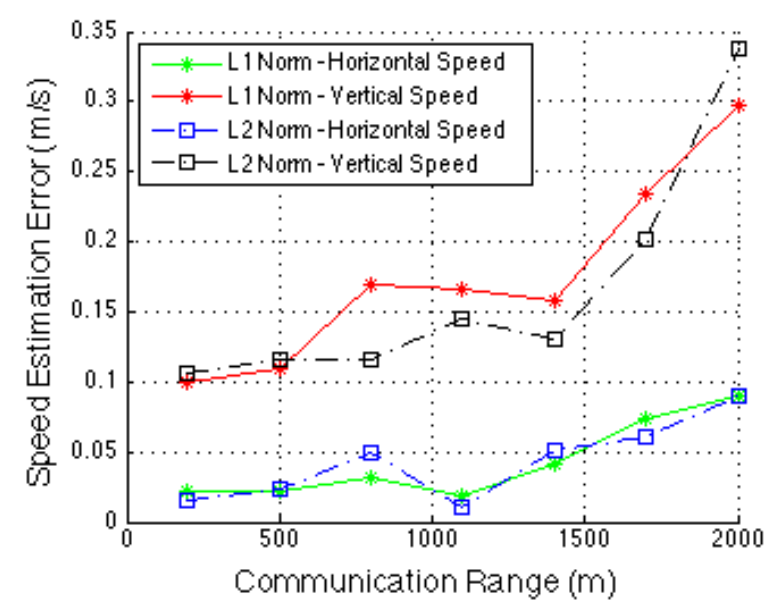

(b)

Fig. 9: Source speed estimation. (a) Simulation results for different source speeds varying from 0 to $3 \mathrm{~m} / \mathrm{s}$. (b) Simulation results for constant source speed ( $2 \mathrm{~m} / \mathrm{s}$ horizontal, $1 \mathrm{~m} / \mathrm{s}$ vertical) and varying communication range from $200 \mathrm{~m}$ to $2 \mathrm{~km}$.

no environmental mismatch in the ray tracer between data generation and computation of predicted delays.

Evaluation of source speed estimation using UWA channel simulator is presented in Figures $9 \mathrm{a}$ and $9 \mathrm{~b}$ applying either $\ell_{1}$ or $\ell_{2}$ norms in (11). Figure 9a provides the estimation error as a function of different source speeds (from 0 to $3 \mathrm{~m} / \mathrm{s}$ ), for horizontal (range) and vertical (depth) velocity components separately. Figure $9 \mathrm{~b}$ presents the same criterion as a function of communication range for constant horizontal speed of $2 \mathrm{~m} / \mathrm{s}$ and vertical speed of $1 \mathrm{~m} / \mathrm{s}$ (varying communication range from $200 \mathrm{~m}$ to $2 \mathrm{~km}$ ). As expected, the horizontal velocity component is more accurately estimated, as in the chosen geometric configurations it has a dominant contribution to the observed Doppler shifts. In all cases less than $10 \%$ estimation error for horizontal speed and a maximum of $15 \%$ error in vertical speed are observed.

To assess the proposed approach for velocity estimation of the acoustic source in 3D, several cases with various arraysource configurations are considered. Figure 10 presents the top view of the channel for all cases with the same structure as in $8 \mathrm{a}$. The red and blue vectors respectively define the actual and estimated velocity vectors. Unlike the presented results for 3D localization, in addition to the dependency on channel configuration and communication ranges, speed estimation errors also depend on the direction of the velocity vector of the transmitter. The actual and estimated velocity vectors and the error for each case are presented in Table I. As the results illustrate, for various cases in $3 \mathrm{D}$, the speed estimation error is highly dependent on the projection of the velocity vector on arrays-source paths. In an ideal case, we achieved 5\% estimation error, which is related to the first case. The worse possible combination for velocity estimation is demonstrated in case 6, with $72 \%$ estimation error. Regardless of the channel configuration, in all cases, the vertical component of the

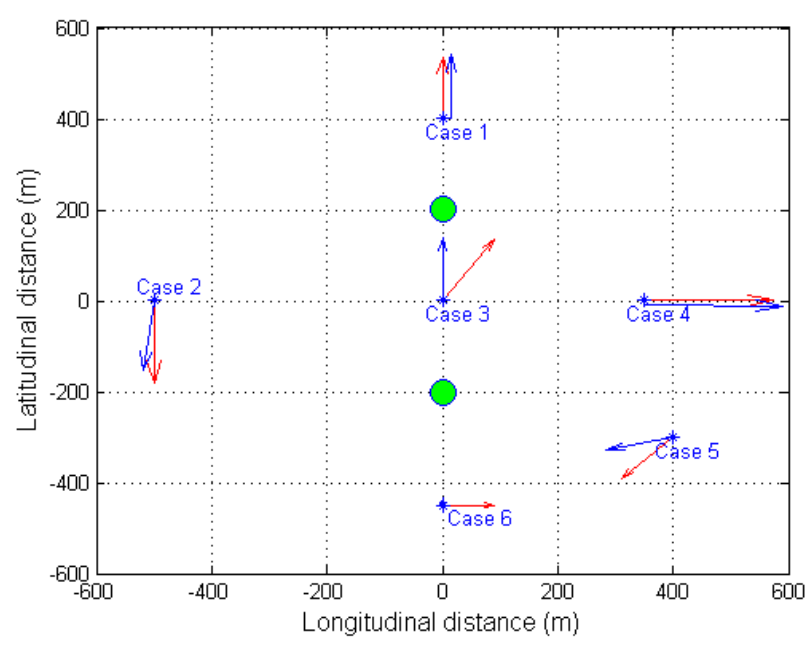

Fig. 10: Evaluation of the velocity estimation for a moving acoustic transmitter in 3D using simulation data for various channel configuration. Top view of the channel with two linear receiver arrays located at $(0,200)$ and $(0,-200)$, defined by by green circles. Red and blue vectors respectively define the actual and estimated velocity vector.

velocity vector is detectable with reasonable estimation error.

\section{CONCLUSION}

This work evaluates the performance of previously proposed acoustic source localization and speed estimation in 3D using two linear receiver arrays. This is intended as a contribution to the development of high-frequency tomographic methods that can be used to extract useful environmental information from communication signals, in addition to the digital messages themselves. 
TABLE I: 3D velocity vector estimation error for different channel configurations.

\begin{tabular}{|c|c|c|c|}
\hline Case No. & $\begin{array}{c}\text { Actual Velocity } \\
\text { Vector }(\mathrm{m} / \mathrm{s})\end{array}$ & $\begin{array}{c}\text { Estimated Velocity } \\
\text { Vector }(\mathrm{m} / \mathrm{s})\end{array}$ & $\begin{array}{c}\text { Estimation } \\
\text { Error }(\%)\end{array}$ \\
\hline \hline Case 1 & $\mathrm{~V}=(0,1.5,1)$ & Vest $=(0,1.57,0.92)$ & $5 \%$ \\
\hline Case 2 & $\mathrm{~V}=(0,-2,1)$ & Vest $=(-0.21,-1.68,1.09)$ & $18 \%$ \\
\hline Case 3 & $\mathrm{V}=(1,1.5,1)$ & Vest $=(0,1.54,1.08)$ & $49 \%$ \\
\hline Case 4 & $\mathrm{V}=(2.5,0,1)$ & Vest $=(2.68,-0.10,1.14)$ & $8 \%$ \\
\hline Case 5 & $\mathrm{V}=(-1,-1,1)$ & Vest $=(-1.19,-0.32,1.15)$ & $41 \%$ \\
\hline Case 6 & $\mathrm{V}=(1,0,1)$ & Vest $=(0,-0.18,0.94)$ & $72 \%$ \\
\hline
\end{tabular}

The 3D localization approach relies on matching predicted and observed arrivals in channel responses obtained at two arrays of receivers. The first part of the localization scheme, termed "coarse source localization", tries to provide a good initial point for source position to be used for the second step, "model based source localization". The results presented in the paper demonstrate that the proposed 3D-CSL method is quite accurate and can be employed as a stand-alone estimator over short communication ranges (below $600 \mathrm{~m}$ ), or when only a rough source position estimate is needed. In order to have a more accurate estimation when reliable environmental information is available, especially for longer communication ranges, the MBSL method can be used.

The methods were successfully tested using simulated data for $2 \mathrm{D}$ and $3 \mathrm{D}$ configurations for different communication ranges and varying relative speed. In simulation, MBSL converged to within $1 \mathrm{~m}$ of the true source position in as few as 2 iterations. However, MBSL is more computationally complex than CSL, as it involves running a ray trace at each iteration. The estimation error for both CSL and MBSL depends on the configuration of the receiver array and the acoustic transmitter.

In addition, as a part of this work, the sensitivity of the MBSL to environmental mismatches such as deviations in measured SSP, bathymetry and receiver array position is assessed. As the results demonstrate, MBSL estimation errors present a predominantly linear trend in response to deviations in sound speed and depth profiles. Besides we can infer that for reasonable mismatches in model inputs, the system output remains in an acceptable region.

The improvement of several processing blocks in our tomographic scheme, e.g., accounting for the strong correlation of channel responses across space to improve the overall estimation efficiency, or introducing some amplitude information in the CSL and MBSL localization schemes to account for the higher reliability of propagation delays estimated from stronger wavefronts, will be evaluated in future work.

\section{ACKNOWLEDGMENTS}

This work was supported by Fundação para a Ciência e a Tecnologia (project PTDC/EEA-CRO/104243/2008 and ISR/IST plurianual funding), and EU project MORPH (grant agreement no. 288704) under the 7th Framework Programme. The authors would like to thank CINTAL/Universidade do Algarve for conducting most of the activities in the CALCOM' 10 sea trial.

\section{REFERENCES}

[1] L. M. Brekhovskikh and Y. P. Lysanov, Fundamentals of Ocean Acoustics. Springer, 1991.

[2] F. B. Jensen, W. A. Kuperman, M. Porter, and H. Schmidt, Computational Ocean Acoustics. New York: American Institute of Physics Press, 1994.

[3] E. Zamanizadeh, J. Gomes, and J. Bioucas-Dias, "Identification of sparse time-varying underwater channels through basis pursuit methods," in Proceedings of the 10th European Conference on Underwater Acoustics (ECUA'10), Istanbul, Turkey, July 2010.

[4] W. Li and J. C. Preisig, "Estimation of rapidly time-varying sparse channels," IEEE Journal of Oceanic Engineering, vol. 32, no. 4, pp. 927-939, October 2007.

[5] P. A. Bello, "Characterization of randomly time-variant linear channels," IEEE Transactions on Communications Systems, vol. CS-11, pp. 360393, December 1963.

[6] E. Zamanizadeh, J. Gomes, and J. Bioucas-Dias, "Source localization from time-differences of arrival using high-frequency communication signals," in OCEANS 2011, Kona, Hawaii, USA, Sep 2011.

[7] A. Beck, P. Stoica, and J. Li, "Exact and approximate solutions of source localization problems," IEEE Transactions on Signal Processing, vol. 56, no. 5, pp. 1770-1778, May 2008.

[8] E. Zamanizadeh, J. Gomes, and J. Bioucas-Dias, "Model-based underwater acoustic source localization and speed estimation using highfrequency transmissions," in Proceedings of the 11th European Conference on Underwater Acoustics (ECUA'12), Edinburgh, UK, July 2012.

[9] X. Ma and Z. H. Michalopoulou, "Matched arrival processing for efficient inversion in underwater acoustics," in Proceedings of MTS/IEEE OCEANS'99, vol. 3, Seattle, USA, September 1999, pp. 1577-1580.

[10] E. Zamanizadeh, J. Gomes, and J. Bioucas-Dias, "Wavefront segmentation and classification for model-based underwater high-frequency tomography," in OCEANS 2012, Virginia Beach, Virginia, USA, Oct 2012.

[11] S. E. Dosso, M. R. Fallat, B. J. Sotirin, and J. L. Newton, "Array element localization for horizontal arrays via Occam's inversion," Journal of the Acoustical Society of America, vol. 104, no. 2, pp. 846-859, 1998. 